\title{
Marcela Venebra MuÑoz: \\ La reforma fenomenológica de la antropología. Bogotá, Editorial Aula de Humanidades/ Universidad de San Buenaventura, 2017, 359 PP.
}

\author{
Noé Expósito Ropero ${ }^{1}$ \\ UNED \\ nexposito@fsof.uned.es
}

La reciente publicación que reseñamos, La reforma fenomenológica de la antropología, supone una contribución fundamental tanto para las investigaciones en Fenomenología como en Antropología -y no solo filosófica, tal y como señala Javier San Martín en su "Prólogo" (p. 22)-, y es que, como advierte Ignacio Quepons en su "Presentación", en ella se parte de los contextos del debate entre ambas disciplinas, enfatizando los diferentes aspectos de la subjetividad trascendental concreta, exponiendo "el programa de ruta hacia una comprensión de la especificidad filosófica de la fenomenología trascendental, como vocación humana" (p. 14). Esta es, efectivamente, la compleja labor que se propone la autora a lo largo de los diez capítulos que componen esta obra, los cuales merecerían por sí mismos un detenido y pormenorizado comentario, tarea que postergamos para otra ocasión y que esperamos poder presentar en el próximo número del Acta Mexicana de Fenomenología (http://actamexicanadefenomenologia.org), dirigida por la propia Marcela Venebra. En lo que sigue nos limitamos a exponer resumidamente las líneas generales de esta obra, así como los temas centrales que en ella se abordan, con la intención de invitar al lector interesado a una confrontación directa con la misma.

\footnotetext{
${ }^{1}$ Beneficiario del Programa de Formación de Profesor Universitario (FPU) 2015-2019 del Ministerio de Educación y Ciencia, adscrito al Departamento de Filosofía y Filosofía Moral y Política de la UNED. Prepara una tesis doctoral sobre la ética fenomenológica de Edmund Husserl y José Ortega y Gasset bajo la dirección del profesor Javier San Martín.
} 
Antes de entrar a comentar el contenido de esta investigación, debe destacarse en ella tanto su rigor textual y metodológico como la claridad y fluidez de su escritura, pues no resulta nada sencillo adentrarse en tan complejas problemáticas sin caer en la oscuridad de los tecnicismos terminológicos, escollo que la autora solventa recurriendo a las fuentes originales decisivas, muy bien escogidas, pero también mediante ejemplos y aclaraciones propias que nos presentan de un modo claro y evidente los puntos centrales que se abordan en cada caso. Del mismo modo, tanto la "Introducción" (pp. 23-39) como el capítulo final, "Radicalización fenomenológica de la antropología" (pp. 315-322), reflejan el esfuerzo de la autora por ofrecernos una síntesis clara y sistemática de las líneas e ideas directrices de su trabajo, así como el inicio de cada capítulo, que recoge y parte directamente de los resultados obtenidos en el apartado precedente, confiriendo así una unidad y progresión a la investigación que contribuyen notablemente a su desarrollo.

Entrando ya en los temas abordados, la autora nos muestra desde la primera página de su "Introducción", partiendo de una cita de Husserl, cuál es la cuestión central a dilucidar, y no es otra que la distinción -en palabras del fundador de la fenomenología - entre "la antropología ingenua que permanece en la positividad [y que es] filosóficamente inauténtica" y "una verdadera antropología filosóficamente auténtica, y es aquella que elucida el sentido absoluto de la existencia humana y la mundanidad, y que legitima a través de un método rigurosamente científico" (p. 23). Ahora bien, entender el trasfondo filosófico de esta problemática, así como las razones últimas que la sustentan, no es tarea fácil, sino que nos exige revisar críticamente tanto las nociones fundamentales de la fenomenología -y su método- como la interpretación "convencional" de la misma.

La relación entre Fenomenología y Antropología ha sido compleja y ambigua desde el comienzo, tanto para Husserl como para sus críticos, de ahí que la autora dedique el primer capítulo del libro al problema de "La carencia antropológica de la fenomenología" (pp. 41-83), haciéndose cargo de los argumentos de Hans Blumenberg contra la posibilidad de una antropología filosófica en general, puesto que, como señala Venebra, para Blumenberg, "la «conversión» antropológica de la fenomenología sólo puede ser una catástrofe" (p. 49). Sin embargo, la autora nos muestra detalladamente que esta "crítica externa" a la fenomenología se basa en una visión parcial y convencional de la misma, entendiéndola 
fundamentalmente como teoría del conocimiento, y desatendiendo las motivaciones del movimiento fenomenológico. Este será, precisamente, un punto central en el que insistirá la autora, pues no sería posible comprender la coherencia y continuidad de la obra de Husserl, desde las Investigaciones lógicas hasta La crisis, pasando por Ideas $I$, sin atender a su vocación ético-práctica de reconstruir una racionalidad universal amenazada por el relativismo, el psicologismo y el antropocentrismo - formas todas ellas de escepticismo. Esta será, efectivamente, la perspectiva lograda y perfectamente justificada en el capítulo nueve del libro, el penúltimo, titulado "El ideario trascendental de la autenticidad racional" (pp. 293-314), en el que Venebra, en diálogo crítico con Robert Sokolowski, mantiene que "La razón, la racionalidad, no es una determinación ontológica del hombre, no es su condición: es su vocación. El hombre es el sujeto que puede aspirar a una vida en la verdad" (p. 295). En consonancia con esta idea, insiste la autora en la intrínseca relación entre "epojé y vocación ética" (pp. 306-308), así como en "La función existencial de la fenomenología trascendental" (pp. 308314), para llegar a la decisiva conclusión de que "la antropología fenomenológica es, también, el necesario fundamento de la ética" (p. 314).

Con lo anterior hemos trazado, grosso modo, las coordenadas del libro, su punto de partida y de llegada, pero si este camino se hubiese recorrido atendiendo únicamente a las "críticas externas" a la fenomenología, estaríamos hablando - a estas alturas de las investigaciones en fenomenología - de un estudio interesante, quizás muy meritorio, pero la profunda Reforma fenomenológica de la antropología que nos propone Venebra consiste, precisamente, en hacerse cargo de la "crítica interna" que el propio Husserl realizó a la antropología -tal y como era entendida en ese momento histórico. Este es el gran valor del libro que nos ocupa, puesto que, tomando esta problemática como hilo conductor, y partiendo de las "Motivaciones históricas de la crítica al antropologismo" (pp. 7983), la autora nos muestra, ya en el segundo capítulo, la estrecha relación entre esta y la crítica a la sofística y al psicologismo, de ahí "la importancia de la vinculación originaria entre la reforma platónica y la reforma husserliana" (p. 93). Ahora bien, una vez que la autora ha trazado el panorama general, mostrando las consecuencias que la pérdida de la validez de la razón universal tiene tanto en el plano teórico como en el ético-práctico, puede adentrarse ya en la problemática interna que articula la fenomenología husserliana, desde la psicología 
pura hasta el problema de la identidad: "Desde la psicología pura y a través de la epojé se abre la esfera trascendental y en el tránsito de uno a otro estrato aparece el problema de la identidad y nos conduce a su tematización en la arquitectónica fenomenológica" (p. 103).

Este será, justamente, el tema central del tercer capítulo, titulado "Identidad de la vida subjetiva y Antropología Filosófica" (pp. 115-124), en el que Venebra nos reconduce hasta "Ese descentramiento localizado en el origen de la vida trascendental", el cual sería "él mismo condición de posibilidad autoconstituyente del yo" (p. 123). Pero, dando un paso más - profundos y cautelosos pasos de gigante que aquí no podemos más que enumerar - el capítulo cuarto se adentra en el estudio del "yo puro" como "polo-yo" -el "yo puro" de las Ideas que "no tiene cuerpo pero se desplaza y se orienta en la corriente de conciencia" (p. 125), señala Venebra-, pasando por la explicitación de "El mundo como correlato trascendental" (pp. 134-139) y "El desplazamiento [Verschiebung] de la epojé fenomenológica" (pp. 139-149), para desembocar, ya el capítulo quinto, en el análisis de la sugerente noción de "carne trascendental" - propuesta por Nathalie Depraz- y su papel en la "objetivación del cuerpo" (pp. 173-183). Ahora bien, antes de arribar a ella, la autora se ocupa en los epígrafes precedentes de este quinto capítulo de otras dos nociones centrales en la fenomenología de Husserl: las "Sensaciones de movimiento: cinestesias" (pp. 160-168) y las "Sensaciones táctiles: ubiestesias" (pp. 168-173). Así, la tesis que nos propone Venebra tras el minucioso análisis de estas problemáticas, es la siguiente: "que la auto-objetivación del cuerpo humano como una instancia del yo, expone un rasgo antropogénico. Solo el cuerpo humano -entre todos los seres animados conocidos fácticamente- sería auto-aprehensible como una objetividad identificable con el yo" ( $p$. 178).

Esta tesis nos lleva, ya en el capítulo sexto, titulado "El yo humano. Vida espiritual", al problema de la relación entre "Descentramiento, autorreflexión e identidad personal" (pp. 185-187), así como al vínculo entre "Conciencia de sí e intersubjetividad" (pp. 187-209), para poder abordar con toda claridad el problema central de la "Vida espiritual como vida motivada" (pp. 209-223), respecto al cual aclara la autora que "Las sedimentaciones más profundas fundan inclinaciones del carácter a las que se pueden llamar intereses" (p. 213), y esto porque "Las habitualidades predelinean una disposición incluso para el estilo de la 
percepción" (p. 212). Todo esto entronca, a su vez, con el papel de la historia en la configuración personal de cada individuo, respecto a lo cual mantiene la autora que, "desde el punto de vista fenomenológico la historia es una forma de conciencia de sí ampliada en un nivel intersubjetivo" (p. 219).

Todo lo anterior cobra pleno sentido cuando, en el capítulo séptimo, Venebra desarrolla la noción decisiva en la fenomenología de Husserl de "persona trascendental", mostrándonos cómo esta es entendida como "concreción trascendental" (pp. 225-252). La idea central esbozada aquí por la autora sería que "La persona trascendental es el descubrimiento de la trascendentalidad $y$, fenomenológicamente, antropológicamente, lo verdaderamente importante está en las condiciones y posibilidades [o potencialidades] de ese descubrimiento" (p. 251). Al hilo de esta cuestión clave matiza la autora, en el último epígrafe de este séptimo capítulo, que "Hay que distinguir en la generatividad la protogeneratividad, en cuyo umbral nos dejan los análisis genéticos" (p. 254), lo cual entronca con la «fenomenología constructiva» planteada por Eugen Fink. Esta "se ocupa de los temas y problemas de la generatividad, que comprende la totalidad de las teorías fenomenológicas que ya no se atienen a lo dado en la intuición" (p. 258), por lo que "tiene entre sus temas las experiencias del nacimiento y la muerte" (p. 259).

Con ello llegamos a uno de los capítulos centrales -y más sugerentes a mi juicio- del libro, el octavo, titulado "Yo fenomenologizante. Vida auténtica" (pp. 265-292), puesto que en él se reúnen todas las problemáticas abordadas en los capítulos precedentes, pero analizadas ahora desde una última vuelta de tuerca al hilo de la "«problemática ulterior y última de la fenomenología - la de la crítica de sí misma-» [en palabras del propio Husserl], la de la teoría trascendental del método y su tematización de la función del espectador fenomenologizante" (pp. 280-281). Y es que, tal y como nos aclara la autora, "La vida trascendental es ella misma una excedencia de la existencia humana, por su trascendentalidad la vida humana es capaz de des-localizarse, de verse a sí misma desde un «preser»", idea introducida por Fink en la Sexta meditación cartesiana para mostrar que "si la vida trascendental es lo que constituye el sentido del ser del mundo, ella no puede ser entendida o aprehendida con las mismas categorías del ser" ( $p$. 269). Tras abordar todas estas complejas problemáticas - sin olvidar la famosa "deshumanización" [Entmenschung] requerida por la epojé-, Venebra rescata la 
siguiente idea central, y que nos interesa resaltar aquí: "No se trata de «dejar» de ser hombre sino en sentido metafórico, pues el acto filosófico «el primero y el último» requiere más bien una profundización de ese sentido humano inmediato, una radicalización de aquello que constituye la cualidad central de la persona humana, su capacidad de auto-conciencia" (p. 290). Esta reflexión nos muestra que incluso la más honda, técnica y compleja problemática que nos plantea la fenomenología de Husserl tiene como trasfondo último al ser humano, de ahí que - para finalizar esta reseña otorgándole a la autora la última palabra - "la reforma fenomenológica de la antropología filosófica se convierte en reforma de la vocación humana de la filosofía toda, se convierte en el retorno al primer acto que aspira a la fundamentación radical de la vida misma" (p. 291). 